\title{
The spatial regulation of condensin activity in chromosome condensation
}

\author{
Rebecca Lamothe, ${ }^{1}$ Lorenzo Costantino, ${ }^{1}$ and Douglas E. Koshland \\ University of California at Berkeley, Berkeley, California 94720, USA
}

\begin{abstract}
Condensin mediates chromosome condensation, which is essential for proper chromosome segregation during mitosis. Prior to anaphase of budding yeast, the ribosomal DNA (RDN) condenses to a thin loop that is distinct from the rest of the chromosomes. We provide evidence that the establishment and maintenance of this RDN condensation requires the regulation of condensin by Cdc5p (polo) kinase. We show that Cdc5p is recruited to the site of condensin binding in the RDN by cohesin, a complex related to condensin. Cdc5p and cohesin prevent condensin from misfolding the RDN into an irreversibly decondensed state. From these and other observations, we propose that the spatial regulation of $\mathrm{Cdc5p}$ by cohesin modulates condensin activity to ensure proper RDN folding into a thin loop. This mechanism may be evolutionarily conserved, promoting the thinly condensed constrictions that occur at centromeres and RDN of mitotic chromosomes in plants and animals.
\end{abstract}

[Keywords: cohesion; condensation; condensin; cohesin; polo kinase]

Supplemental material is available for this article.

Received November 27, 2019; revised version accepted March 27, 2020.

Mitotic chromosomes have two structural features, sister chromatid cohesion and chromosome condensation, which are essential for their proper inheritance during cell division. Sister chromatid cohesion is established concurrently with DNA replication and is maintained until the onset of anaphase. In contrast, condensation is established in mitotic prophase and persists through anaphase until the end of mitotic division. As a result, cohesion and condensation coexist from prophase until the onset of anaphase, a period referred to here as mid-M. Perturbing proper condensation or cohesion leads to aneuploidy and chromosome rearrangements, hallmarks of cancer, birth defects, and several genetic disorders (Hassler et al. 2018). Over the last two decades, scientists have made significant progress in identifying the protein complexes that mediate cohesion and condensation and understanding their cell cycle regulation. However, the potential interplay between these complexes has remained largely uninvestigated.

Cohesion and condensation are mediated by cohesin and condensin, respectively, two related protein complexes in the SMC (structural maintenance of chromosomes) family (Fig. 1A; Hassler et al. 2018). Cohesin intermolecularly tethers DNA of the sister chromatids, resulting in cohesion, while condensin promotes condensation by tethering DNA intramolecularly. Condensin is also thought to promote condensation by inducing supercoils

\footnotetext{
${ }^{1}$ These authors contributed equally to this work.

Corresponding author: koshland@berkeley.edu

Article published online ahead of print. Article and publication date are online at http://www.genesdev.org/cgi/doi/10.1101/gad.335471.119.
}

and by actively extruding DNA to form loops (Kimura and Hirano 1997; Kimura et al. 2001; Hagstrom et al. 2002; St-Pierre et al. 2009; Ganji et al. 2018). In principle, the existence of distinct SMC complexes dedicated to cohesion and condensation would allow these processes to occur independently. Consistent with this conclusion, mammalian chromosomes can condense in the absence of cohesion (Losada et al. 1998; Sonoda et al. 2001).

The independence of cohesion and condensation also seems to be supported by studies of their regulation. The establishment of cohesion in S phase is regulated by Ecolp-dependent acetylation of cohesin (Rolef Ben-Shahar et al. 2008; Ünal et al. 2008), while its maintenance is mediated by interactions with Pds5p, a cohesin auxiliary factor (Noble et al. 2006). In contrast, the phosphorylation of condensin by different kinases mediates the establishment and maintenance of condensation. Cyclin-dependent kinases (CDKs) are required to establish condensation in prophase (Kimura et al. 1998). Polo kinase and Aurora kinase are important for the maintenance of condensation after the onset of anaphase when CDK activity diminishes (Lavoie et al. 2002, 2004; St-Pierre et al. 2009; Robellet et al. 2015). These observations suggest that cohesion and condensation are mediated by distinct protein complexes with distinct regulatory circuits. However, cohesin inactivation abrogates condensation as

(C) 2020 Lamothe et al. This article is distributed exclusively by Cold Spring Harbor Laboratory Press for the first six months after the full-issue publication date (see http://genesdev.cshlp.org/site/misc/terms.xhtml). After six months, it is available under a Creative Commons License (Attribution-NonCommercial 4.0 International), as described at http://creativecommons.org/licenses/by-nc/4.0/. 
Lamothe et al.

A

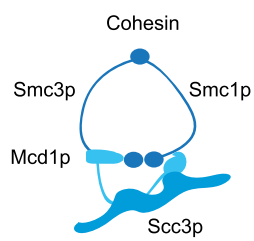

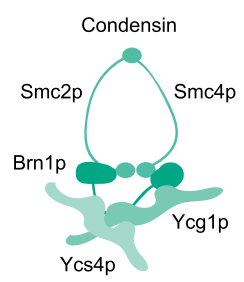

D
B

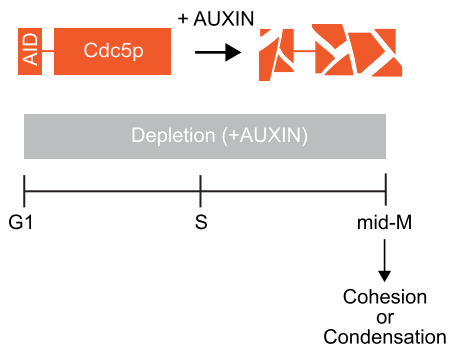

C

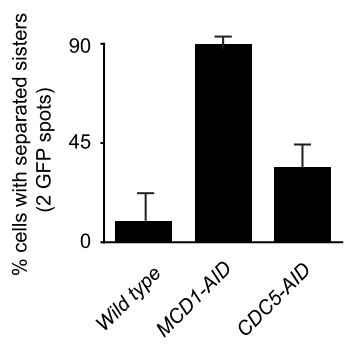

$\mathbf{E}$
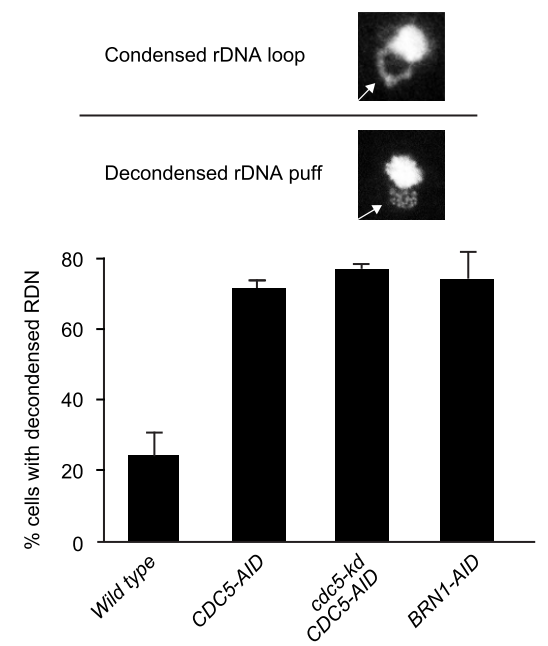

$\mathbf{F}$
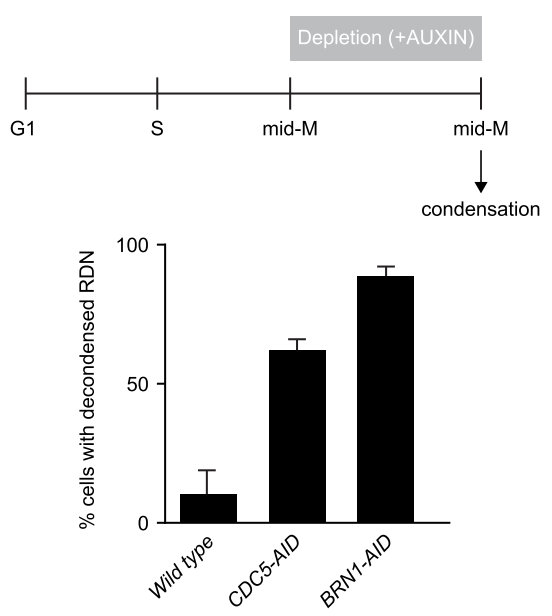

Figure 1. The polo kinase Cdc5p is essential for mid-M chromosome condensation. $(A)$ Schematic of cohesin and condensin. Cohesin is comprised of a Smc1p/Smc3p heterodimer joined at their head domain through Mcdlp. Mcdlp is likewise bound to Scc3p, resulting in the cohesin holocomplex. Condensin is comprised of a Smc2p/Smc4p heterodimer linked by Brn1p. Brn1p is bound to Ycglp and Ycs4p. Together, these proteins form the pentameric condensin complex. $(B$, top $)$ Schematic of the auxin-induced degron (AID) system to study functions of essential proteins. Proteins of interest, like Cdc5p, were fused to a 3V5-AID tag that allows for wild-type functions in the absence of auxin. Upon addition of auxin, 3V5-AID-tagged proteins were rapidly degraded in a proteasome-mediated manner. (Bottom) "Staged mid-M" regimen used to assess strains function in mid-M. Cultures were synchronized in G1 and released in media containing auxin and nocodazole, to depleted AID-tagged protein and arrest cells in mid-M. $(C)$ Depletion of Cdc5p results in a moderate cohesion defect. Cells were treated as in 1B and cohesion assayed in mid-M through a GFP spot assay. In these experiments, LacO arrays are integrated at LYS4, a centromere-distal arm site. Wild-type, Mcd1p-depleted, and Cdc5p-depleted cells were scored for sister chromatid cohesion. The average percentage of separated sister chromatids from three independent experiments (100 cells) are reported, and error bars represent standard deviation. $(D)$ Depletion of Cdc5p results in a moderate cohesion maintenance defect. Wild-type, Pds5p-depleted, Cdc5p-depleted, and Cdc5 kinase-dead (kd)-expressing in combination with depletion of Cdc5p-AID cells were treated as in $B$ and cohesion assayed at 15-min intervals after release from G1 to mid-M. The average percentage of separated sister chromatids from two independent experiments (100 cells) are reported. $(E)$ Depletion of Cdc5p results in condensation loss. (Top) Example of rDNA locus (RDN) morphologies assayed. RDN is either assayed as a condensed loop or decondensed puff. The percentage of cells displaying decondensed RDN puffs is quantified. (Bottom) Wild-type, Cdc5p-depleted, Cdc5 kinase-dead (kd)-expressing in combination with depletion of Cdc5p-AID, and Brnlp-depleted cells were treated as in $B$ and processed to make chromosome spreads to score RDN condensation (see the Materials and Methods). Average of three experiments scoring 200 cells are shown and error bars represent the standard deviation. $(F)$ Depletion of Cdc5p results in loss of maintenance of condensation. (Top) Cells were arrested in mid-M using nocodazole. The arrest was confirmed by analysis of bud morphology ( $>95 \%$ large budded cells). Auxin was added to ensure Cdc5p-AID degradation. (Bottom) Wild-type, Cdc5p-depleted, and Brn1p-depleted cells were processed to assess condensation as described in $E$. 
well as cohesion in mitosis of budding yeast, meiosis of fission yeast, and mouse oocytes, and sperm /Guacci et al. 1997b; Ding et al. 2006; Novak et al. 2008; Guacci and Koshland 2012). A subsequent study in budding yeast showed that cohesin is not required for condensation per se, but rather prevents condensin from permanently misfolding chromosomes into an irreversibly decondensed state (Lavoie et al. 2002). How cohesin regulated condensin in mid-M remained a mystery.

A candidate for mediating this regulation was polo kinase. In budding yeast, polo kinase (Cdc5p) binds to cohesin-associated regions (CARs) on chromosomes, and the binding is dependent upon cohesin (Pakchuen et al. 2016). Cdc5p also is required for mid-M condensation and phosphorylates condensin in mid-M (St-Pierre et al. 2009; Walters et al. 2014; Archambault et al. 2015; Pakchuen et al. 2016). Here we show that Cdc5p is required for both the establishment and maintenance of condensation of the 1-Mb ribosomal DNA (RDN) in mid-M, likely by promoting condensin activity to make chromosome folds. We provide additional evidence that suggests cohesin recruits Cdc5p to the RDN, facilitating the activation of adjacently bound condensin. This spatial and temporal activation of condensin promotes RDN condensation into a thin loop and prevents condensin-mediated RDN misfolding.

\section{Results}

The polo kinase Cdc5p is essential for mid-M chromosome condensation

The phenotypes of the different temperature-sensitive cdc5 alleles vary significantly (St-Pierre et al. 2009; Walters et al. 2014). With this in mind, we generated a more stringent loss-of-function cdc5 allele to study the role of $\mathrm{Cdc} 5 \mathrm{p}$ in cohesion and condensation. To assess how essential is Cdc5p function in these processes, we compared Cdc5p depletion with depletion of cohesin and condensin. We took advantage of the auxin-induced degron (AID) system to generate a cdc5 allele (Cdc5p-AID) (Nishimura et al. 2009). Briefly, in this system the fusion of Cdc5p with an AID tag allows for near wild-type function in the absence of auxin. However, in the presence of auxin, tagged proteins are targeted by the E3 ligase TIR1 for rapid ubiquitin-dependent degradation (Fig. 1B, top). We used the AID system to also deplete cohesin (McdlpAID) and condensin (Brn1-AID). After depletion, the AID proteins were undetectable by Western blot and all strains were inviable (Supplemental Fig. S1). Indicated yeast strains were first synchronized in G1, then released into media with auxin to deplete the AID-tagged protein and nocodazole to arrest cells in mid-M (Fig. 1B, bottom). Using this protocol (referred to as staged mid-M) cells were depleted of the AID-tagged protein from G1 to mid-M. We proceeded to assess sister chromatid cohesion and condensation.

We measured sister chromatid cohesion using a wellcharacterized GFP dot assay (Straight et al. 1996). In this system, a lacO array is integrated at a chromosomal locus and bound by lacI-GFP. If proper sister chromatid cohesion is present, the two sister chromatids are held together and the two arrays appear as one GFP spot. Conversely, if cohesion is absent, two distinct GFP spots are observed (Guacci et al. 1997a; Michaelis et al. 1997). We tested wild-type, Cdc5p-AID-depleted, and Mcdlp-AIDdepleted cells. Mcdlp depletion served as positive control for the abrogation of cohesion. Cells lacking Cdc5p-AID had a threefold decrease in sister chromatid cohesion compared with wild-type cells (Fig. 1C). This defect was significantly less than the ninefold decrease in cohesion in cells depleted for Mcdlp-AID. Thus, Cdc5p is important-but not essential-for sister chromatid cohesion.

We performed a time-course experiment for cohesion to assess whether Cdc5p functions in the establishment or maintenance of cohesion. Depletion of factors that are important for the establishment of cohesion resulted in a high level of separated sister chromatids from the end of S-phase till mid-M. While depletion of factors that are involved in the maintenance of cohesion resulted in a gradual loss of cohesion that starts at the end of S-phase and peaks at mid-M (Jin et al. 2009; Eng et al. 2014). We assessed cohesion on wild-type, Cdc5p-depleted, and Pds5p-depleted cells and assayed cohesion at 15-min intervals from G1 to mid-M. Pds5p is a factor required for the maintenance of sister chromatid cohesion after $\mathrm{S}$ phase. Under this regimen, Cdc5p-AID depleted cells presented a weaker defect in cohesion maintenance compared with cells depleted of Pds5p-AID (Fig. 1D). We concluded that Cdc5p is not involved in establishing cohesion but is partially required for cohesion maintenance. Furthermore, cells that expressed a kinase-dead version of Cdc5p in combination with depletion of the endogenous copy of Cdc5p did not present the maintenance defect and were indistinguishable from wild type (Fig. 1D). Therefore, the partial requirement of Cdc5p in cohesion maintenance is independent of its kinase activity.

Using the same regimen described above, we also interrogated the role of Cdc5p in mid-M chromosome condensation by assessing the morphology of the $\sim 1.4-\mathrm{Mb}$ ribosomal DNA model locus (RDN). The RDN contains 75-100 tandem repeats of the 9.1-kb rDNA and undergoes a series of distinct, cell cycle-dependent morphological changes (Fig. 1E, top; Guacci et al. 1994). In interphase, the RDN is a puff that is segregated to the periphery of the bulk chromosomal mass. In contrast, in mid- $M$, the RDN forms a condensed loop. This dramatic cytological RDN change is a tractable assay for condensation. In contrast, the rest of the genome only undergoes twofold compaction that is only measurable by laborious FISH or GFP assays (Guacci et al. 1994; Vas et al. 2007). In mid-M-arrested wild type, $\sim 25 \%$ of cells fail to condense the RDN. In contrast, $\sim 75 \%$ of Cdc5p-AID depleted cells failed to condense the RDN similarly to depletion of a core condensin subunit, Brnlp-AID (Fig. 1E, bottom; Supplemental Fig. S2A). Therefore, Cdc5p-AID depletion caused a severe condensation defect. Ectopic expression of a kinase-dead Ccd5p did not rescue the condensation defect of cells depleted for Cdc5p-AID. Thus, in contrast to cohesion, 
Cdc5p and its kinase activity play a crucial role in RDN condensation in mid-M.

To assess the role of Cdc5p in the establishment of condensation, we performed a time course following RDN condensation in Cdc5p-AID-depleted cells. Ninety minutes is the earliest time point after release from G1 where we could observe condensed RDN loops. Cells depleted for Cdc5p did not establish condensation at $90 \mathrm{~min}$ or later (Supplemental Fig. S2B). Instead, control cells depleted for the maintenance factor Pds5p showed condensed loops at $90 \mathrm{~min}$ that were lost over time. Therefore, Cdc5p is required for the establishment of condensation. To assess whether Cdc5p also functions in condensation maintenance, we modified the auxin regiment of CDC5$A I D$ cells. We first arrested them in mid-M by adding nocodazole, allowing RDN condensation to occur (Supplemental Fig. S3) and then we added auxin to deplete Cdc5p-AID (Fig. 1F, top). We observed a dramatic loss of RDN condensation in cells depleted of Cdc5p-AID in mid-M like that observed in cells depleted for Brn1pAID (Fig. 1F, bottom). Therefore, Cdc $5 \mathrm{p}$ is an essential regulator for the establishment and maintenance of condensation in mid-M.

\section{Cdc5p stimulates condensin-dependent RDN condensation}

How does Cdc5p regulate condensation? Insights come from further examining the RDN condensation defects. Puffed RDN in mid-M can result from either an unfolded state such as is normally seen in G1 or from a misfolded state (Lavoie et al. 2002). The unfolded state is still competent to be folded, while the misfolded state is irreversibly trapped in a decondensed manner (Fig. 2A). These two states were previously distinguished using a simple genetic test referred to here as add later test (Fig. 2B, top; Lavoie et al. 2002). Briefly, temperature-sensitive condensation factors were inactivated from G1 to mid-M preventing condensation, then antiactivated in mid-M, and the reversibility of the condensation defect was assessed. With this test, we showed delayed activation of condensin restores RDN condensation, while delayed activation of cohesin did not (Fig. 2B, bottom; Lavoie et al. 2002). Therefore, condensin inactivation results in an unfolded state, while cohesin inactivation results in an irreversibly misfolded state.

Cdc5p depletion can result in an unfolded state like condensin, or a misfolded state like cohesin. We performed the add later experiments using our AID system to deplete Mcd1p-AID, Brn1p-AID, and Cdc5p-AID (Supplemental Fig. S4A). As expected, depletion of any of these three proteins between G1 and mid-M prevented the appearance of folded RDN (Fig. 2C, gray bars). When Cdc5p-AID was expressed in Cdc5p-AID-depleted cells, the RDN condensed (Fig. 2C, blue bars). The same result was observed when Brn1p-AID was expressed in Brn1p-AID-depleted cells. Conversely, when Mcd1p-AID was expressed in Mcd1pAID-depleted cells, the RDN remained decondensed. These results show that depletion of Cdc5p-AID, like depletion of condensin, results in a reversible unfolded
RDN state, rather than irreversibly misfolded like depletion of cohesin.

We hypothesized that the RDN misfolding that occurred after cohesin depletion was condensin and Cdc5p dependent. If this model was correct, misfolding of the RDN due to cohesin depletion could be prevented by coinactivation of condensin or any factor that stimulates condensin activity. To test this prediction, we depleted and added later two components together (Supplemental Fig. S4A). When we codepleted and added later cohesin and condensin we restored condensation, thus preventing RDN misfolding caused by the absence of cohesin alone (Fig. 2D; Lavoie et al. 2002). When we depleted and added later cohesin and Cdc5p-AID together, we also prevented RDN misfolding caused by the absence of cohesin (Fig. 2D). To explain these genetic results, we propose that Cdc5p is a factor that stimulates condensin activity. When cohesin is present, Cdc5p stimulates condensin to promote proper RDN folding, generating condensation. When cohesin is missing, Cdc5p inappropriately stimulates condensin resulting in a misfolded decondensed RDN.

This model predicts that Cdc5p should phosphorylate condensin with or without cohesion, but the pattern should change in the absence of cohesin. To test this model we staged cells in mid-M with or without cohesin. We measured the level of phosphorylation of the condensin subunit Ycglp, which possesses the highest number of Cdc5p phospho-sites (St-Pierre et al. 2009), using a Phostag gel that slows the migration of phosphorylated proteins. Phosphorylated condensin was observed both in the presence or absence of cohesin as predicted (Supplemental Fig. S4B). Furthermore, the phosphorylation pattern was different in the absence of cohesin: We observed a higher amount of phosphorylated condensin that migrated slower, indicating either more sites being phosphorylated and/or altered phosphorylation pattern. These patterns of Ycg1 phosphorylation are consistent with our model that cohesin is required to achieve proper phosphorylation of condensin.

\section{Cohesin-dependent recruitment of Cdc5p to the RDN promotes its binding to condensin in the RDN}

To dissect the molecular mechanism for how Cdc5p, cohesin, and condensin regulate condensation, we examined their genomic localization by ChIP-seq. We analyzed the binding of Cdc5p, cohesin, and condensin in wild-type and Cdc5p-, cohesin-, and condensin-depleted cells. We also performed an important ChIP-seq control in yeast by immunoprecipitating GFP from GFP-expressing cells. This ChIP-seq provided us with the background signal of genomic regions prone to entrapment by ChIP-seq in the absence of a real signal (Teytelman et al. 2013).

Using the staged mid- $M$ regimen, we first assessed the binding of condensin (Ycglp-Myc), cohesin (Mcd1p), and Cdc5p (Cdc5p-Flag) in wild-type cells (Fig. 3). We observed Ycglp enrichment in a large pericentric region peaking at centromeres and within the end of the nontranscribed region of the RDN (NTS1), corroborating previous results 
A
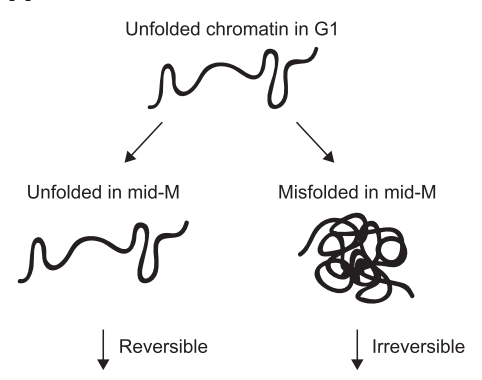

Folded in mid-M

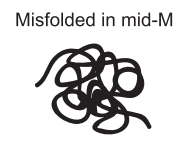

B
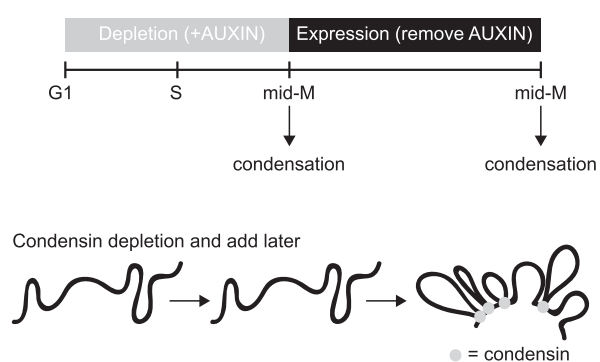

Cohesin depletion and add later

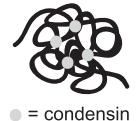

C

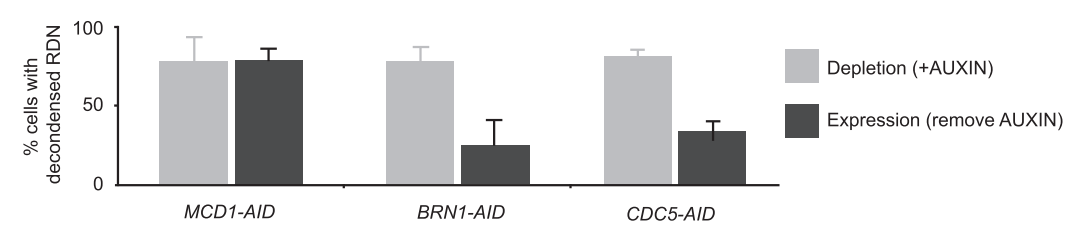

D
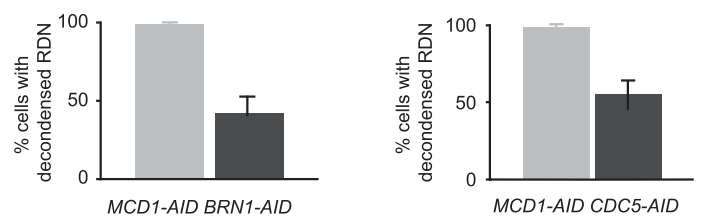

Depletion (+AUXIN)

Expression (remove AUXIN)

Figure 2. Cdc5p stimulates condensin-dependent RDN condensation. (A) Schematic of defects in condensation. RDN is in a puffed unfolded state in G1. Depletion of condensation factors results in a puffed $\mathrm{RDN}$ that can either be in an unfolded state or in a misfolded state. The unfolded state is reversible, once the factor is expressed again the RDN can be folded. Misfolded state is irreversible, even after expressing again the condensation factor the RDN remains misfolded. $(B)$ Schematic of "add lat$\mathrm{er}^{\prime \prime}$ genetic test setup. (Top) Indicated cultures were arrested in G1 (>95\% schmooed morphology), and AID-tagged proteins (Mcdlp-AID, Brnlp-AID, and Cdc5p-AID) were depleted by the addition of auxin. G1 arrest was relieved by resuspending in media with auxin and nocodazole to arrest cells in mid-M. Cells were then resuspended in media containing only nocodazole for $2 \mathrm{~h}$ to allow the expression of the previously depleted AID-tagged proteins. (Bottom) Depletion of condensin resulted in unfolded RDN that was folded upon later expression of condensin. Depletion of cohesin resulted in misfolded RDN that stayed misfolded upon later expression of cohesin. $(C)$ Cdc5p depletion results in a reversible unfolded RDN state. "Add later" genetic test results from $M C D 1-A I D$, $B R N 1-A I D$, and CDC5-AID strains were treated as described in $B$ and scored for $\mathrm{RDN}$ condensation. The percentage of cells displaying puffed RDN (decondensed) is quantified. Average of two experiments scoring 300 cells are shown and error bars represent standard deviation. $(D)$ Condensin and Cdc5p promote misfolding of the RDN in the absence of cohesin. "Add later" genetic test results from MCD1-AID/BRN1-AID and MCD1-AID/CDC5-AID strains were treated as described in $B$ and scored for RDN condensation. The percentage of cells displaying decondensed puffed RDN (decondensed) is quantified. Average of three experiments scoring 300 cells is shown and error bars represent standard deviation.

(Fig. 3; Leonard et al. 2015). Additional peaks of enrichment for Ycg1-Myc that were reported previously (D'Ambrosio et al. 2008) were also observed along the chromosome arms, but they were likely false positives because they coincided with peaks in GFP-control (Fig. 3A). Mcdlp was bound to centromeres, pericentric regions, cohesin-associated regions (CARs) along chromosome arms, and a region between the two nontranscribed spacers (NTS1 and NTS2) in the rDNA repeat (Fig. 3; Laloraya et al. 2000; Glynn et al. 2004; Pakchuen et al. 2016). Finally, the binding pattern of Cdc5p-Flag mirrored cohesin binding genome-wide (Fig. 3; Rossio et al. 2010; Pakchuen et al. 2016). Importantly, we showed that Cdc5p colocalizes with cohesin on the RDN.

We then examined Brnlp-, Mcdlp-, and Cdc5p-binding patterns after depletion of Cdc5p-AID, Brnlp-AID, or Mcdlp-AID. We depleted the relevant factor from G1 to mid-M using the staged mid-M regimen. The depleted factor could not be detected by Western blot. The chromosomal binding pattern of Ycglp-Myc did not change upon the depletion of either cohesin or Cdc5p (Supplemental Figs. S5-S7). Similarly, the binding pattern of
Mcdlp remained unchanged upon depletion of either condensin or Cdc5p (Supplemental Figs. S5-S7). Thus, cohesin and condensin bind to specific sites on chromosomes independently of each other and Cdc5p. Therefore, cohesin and Cdc5p do not regulate RDN condensation by changing condensin localization to the RDN.

We then analyzed whether changes in the localization of Cdc5p might provide insights into RDN condensation. We examined cells in which Mcdlp-AID or Brnlp-AID were depleted from G1 to mid-M using the staged mid$M$ regimen. Upon Mcdlp-AID depletion, the binding of Cdc5p was abolished genome-wide (Supplemental Figs. S5-S7), despite similar levels of total Cdc5p (Supplemental Fig. S8). This result recapitulated previous work showing the cohesin-dependent binding of Cdc5p at CARs (Mishra et al. 2016; Pakchuen et al. 2016). A few small peaks of binding were observed at centromere and RDN regions, but they coincided with false positives in the GFP-control. In contrast, upon Brnlp-AID depletion, the pattern of Cdc5p was largely unaffected genome-wide (Supplemental Figs. S5-S7). These results showed that the recruitment of Cdc5p to chromosomes (including 
A

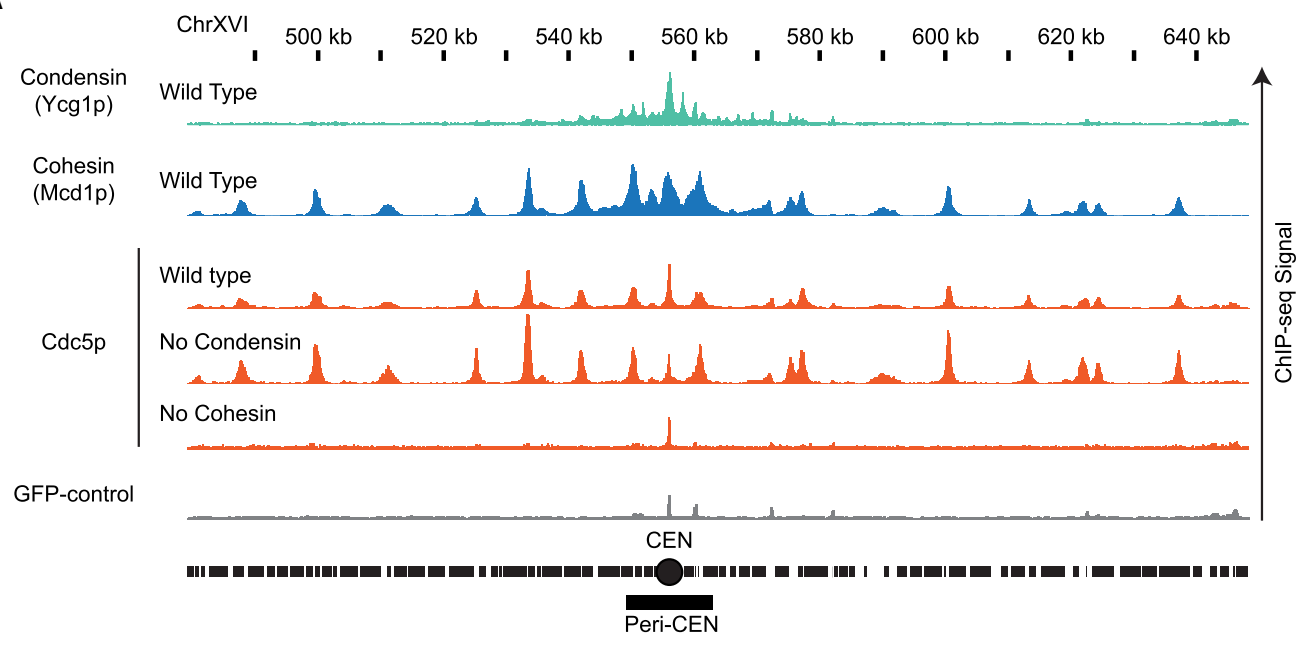

B

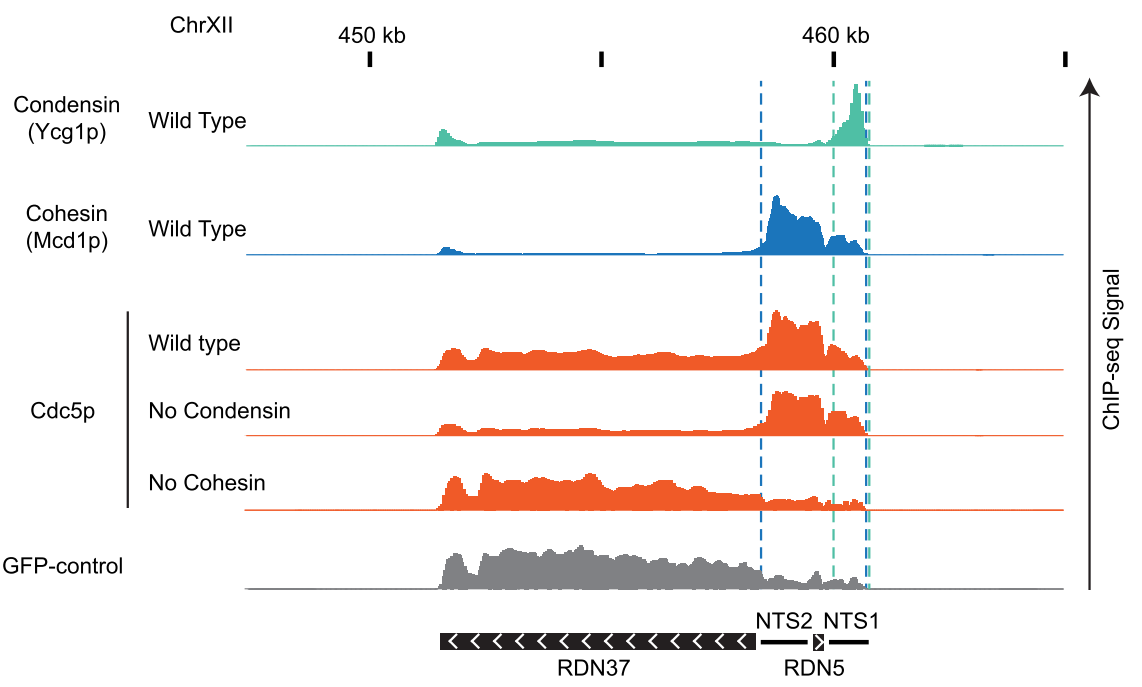

Figure 3. Genome-wide localization of condensin, cohesin and Cdc5p. $(A)$ Cohesin recruits Cdc5p at unique sequences genome-wide. ChIP-seq signals from a representative portion of chromosome XVI are presented with bar charts. The region presented includes the centromere (CEN; black circle) and the pericentric region (peri-CEN; black box). Signal coming from condensin subunit Ycglp-Myc in wildtype strain is in green, signal from cohesin subunit Mcdlp in wild-type strain is in blue, signal coming from Cdc5p-Flag is in orange, and signal coming from GFP-control is in gray. Cdc5p ChIP-seq signal from wild type is in the first orange lane, from cells depleted of condensin subunit Brnlp-AID is in the second orange lane, and from cells depleted of cohesin subunit Mcdlp-AID is in the third orange lane. All strains were depleted of the indicated AID protein from G1 to mid-M and arrested in mid-M (staged mid-M). The scale is 0-15 for Ycg1p, 0-21 for Mcd1p, and 0-17 for Cdc5p. $(B)$ Cohesin recruits Cdc5p at the repetitive RDN locus. ChIP-seq signals from a portion of chromosome XII are presented with bar charts. ChIP-seq signal from strains as in $A$. The region presented includes one copy of the rDNA repeat $(9.1 \mathrm{~kb})$ made of the transcribed regions RND37 and RND5 (black box with white arrows indicating the direction of transcription) and the nontranscribed regions NTS1 and NTS2 (black line). Region of enrichment of cohesin signal is marked by two blue dashed lines (from NTS2 to NTS1). Region of enrichment of condensin signal is marked by two green dashed lines (end of NTS1). The scale is 0-5200 for Ycg1p, 0-2300 for Mcd1p, and 0-1700 for Cdc5p.

the RDN) required the presence of cohesin over some period between G1 to mid-M.

We reasoned the purpose of cohesin recruitment of Cdc5p was to promote localized Cdc5p binding and stimulation of condensin. Therefore, we predicted that a transient association of cohesin with the RDN would be sufficient to bring some Cdc5p to condensin. To test this possibility, we synchronized MCD1-AID cells in mid-M in the absence of auxin to allow cohesin, condensin, and
Cdc5p to normally localize to the RDN. We then added auxin to deplete Mcd1p-AID and remove cohesin. We observed that Cdc5p was eliminated throughout the genome except at a portion of the condensin-bound regions of the RDN and centromeres (Fig. 4; Supplemental Fig. S9). In these regions, we observed peaks of Cdc5p binding that were threefold to fivefold greater than those observed when Mcd1p-AID was removed from G1 to mid-M. The remaining portion of the Cdc5p binding when Mcd1p-AID 
A

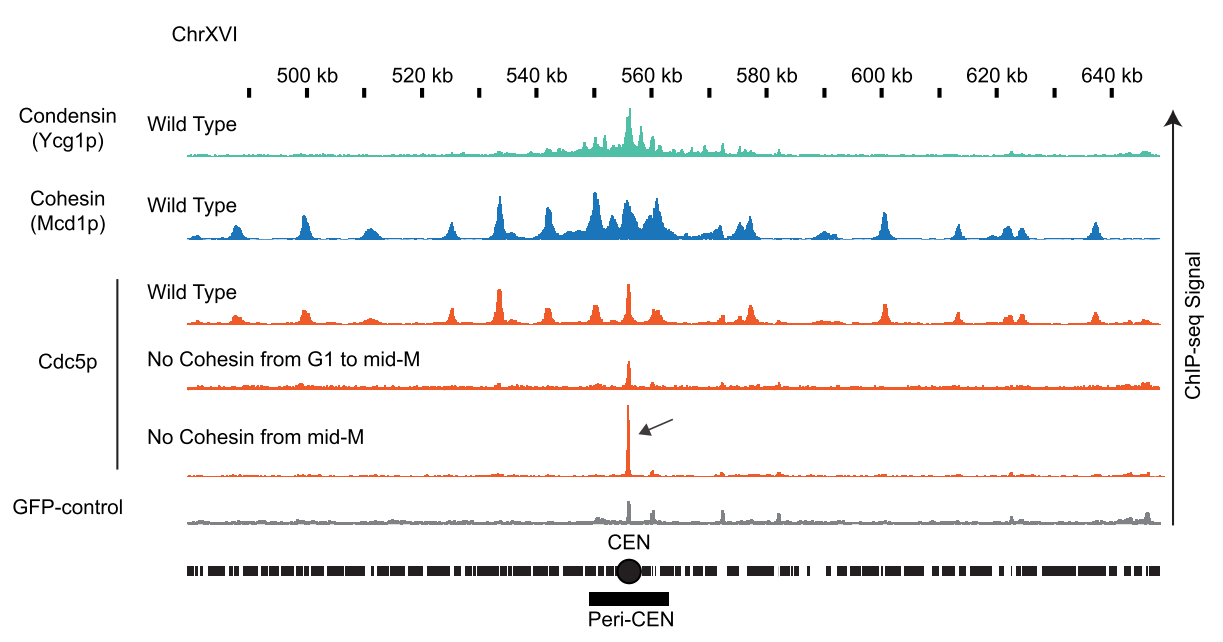

B

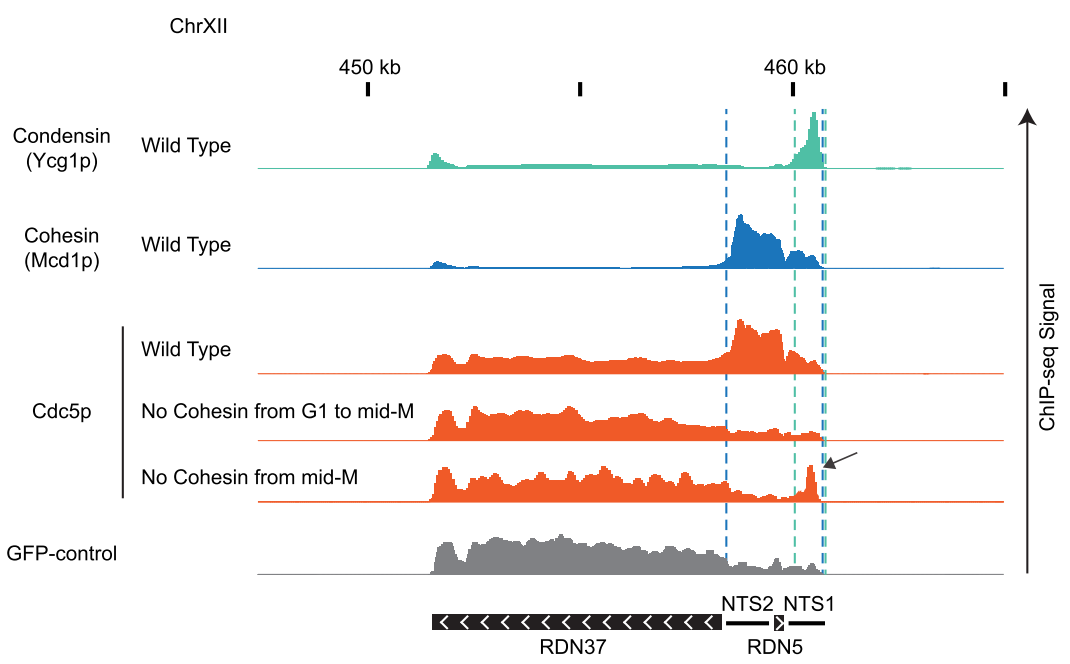

Figure 4. Cdc5p is recruited by cohesin and brought to condensin at centromeres and rDNA. $(A)$ Cdc5p is recruited by cohesin and brought to condensin at centromeres. ChIP-seq signals from a representative portion of chromosome XVI are presented with bar charts. The region presented includes the centromere (CEN black circle) and the pericentric region (peri-CEN black box). Signal coming from condensin subunit Ycglp-Myc in wild-type strain is in green, signal from cohesin subunit Mcdlp in wild-type strain is in blue, signal coming from Cdc5p-V5 is in orange, and signal coming from GFP-control is in gray. Cdc5p ChIP-seq signal from wild type is in the first orange lane, from cells depleted of cohesin subunit Mcd1p-AID from G1 to mid-M is in the second orange lane, and from cells depleted of cohesin subunit Mcd1p-AID in mid-M is in the third orange lane. The black arrow indicates enrichment of Cdc5p at the centromere when cohesin is transiently associated with chromosomes. The scale is $0-15$ for Ycglp, $0-21$ for Mcdlp, and 0-17 for Cdc5p. (B) Cdc5p is recruited by cohesin and brought to condensin at RDN. ChIP-seq signals from a portion of chromosome XII are presented with bar charts. ChIP-seq signal from strains as in $A$. The region presented includes one copy of the rDNA repeat $(9.1 \mathrm{~kb})$ made of the transcribed regions RND37 and RND5 (black box with white arrows indicating the direction of transcription) and the nontranscribed regions NTS1 and NTS2 (black line). Region of enrichment of cohesin signal is marked by two blue dashed lines (from NTS2 to NTS1). Region of enrichment of condensin signal is marked by two green dashed lines (end of NTS1). Black arrow indicates enrichment of Cdc5p at the condensinbound region of the RDN when cohesin is transiently associated with chromosomes. The scale is $0-5200$ for Ycglp, 0-2300 for Mcd1p, and 0-1700 for Cdc5p.

was depleted in mid-M was not sufficient to promote proper condensation (Supplemental Fig. S10). To corroborate our ChIP-seq observations, we used ChIP followed by quantitative PCR (ChIP-qPCR) to follow the temporal dissociation of Cdc5p from the RDN at different time points after cohesin depletion in mid-M (Supplemental Fig. S11). We observed that Mcdlp binding disappeared from the
RDN after 15 min with auxin. Consequently, Cdc5p binding also disappeared from the RDN, except at the site where condensin binds. Here, more than $50 \%$ of Cdc $5 p$ binding persisted, eventually dissipating to background level (90 min). Thus, as suggested from the ChIP-seq, transient Mcdlp binding to the RDN in mid-M is required for Cdc5p binding to the site of condensin binding. We 
propose that in wild type, high levels of cohesin at the RDN bring high levels of Cdc $5 p$ to neighboring condensin, promoting condensin activation and proper condensation.

\section{Localization of Cdc5p with CRISPR-Cas9 \\ to the RDN partially suppresses the \\ condensation defect of cohesin-depleted cells}

If cohesin localization of Cdc5p to condensin at the RDN did indeed promote condensation, this function might be mimicked by ectopic localization of Cdc5p. To test this possibility, we engineered a strain harboring a fusion gene of Cdc5p and catalytically dead dCas9 under the control of the galactose promoter (Fig. 5A). The fusion protein supported the growth of yeast as the sole source of Cdc5p (Supplemental Fig. S12), indicating that this fusion protein retained activity. The strain with the fusion gene into MCD1-AID cells was transformed with plasmids containing either a guide RNA targeting the region of condensin binding at the RDN (NTS1 gRNA) or a distal guide RNA targeting 500 bp away (RDN5 gRNA) (Fig. 5A). The successful target of the fusion to these two sites was corroborated by ChIP-qPCR (Supplemental Fig. S13A). These strains were subjected to the staged mid-M regimen to deplete Mcd1p-AID. The expression of dCas9-Cdc5p was induced from G1 by the addition of galactose to the media, and the ability of the targeted fusion to suppress RDN misfolding was assessed (Fig. 5B, top).

In Mcdlp-depleted cells, only $6 \%$ of the cells show normal condensed RDN loops (Fig. 5B). In contrast, in the presence of guide RNA proximal to condensin, we observed $17 \%$ of cells with normally condensed loops, in addition to $6 \%$ partially condensed (Fig. 5B; Supplemental Fig. S13B). The suppression of RDN misfolding could have been caused by the localization of Cdc5p immediately proximal to condensin, localization anywhere in the RDN, or by the excess of dCas9-Cdc5p fusion protein caused by galactose-induced overexpression. To distinguish between these possibilities, we examined RDN condensation when the overexpressed fusion was targeted $500 \mathrm{bp}$ from condensin binding. No restoration of RDN condensation was observed, demonstrating that neither localization of the fusion anywhere in the RDN nor excess of the fusion protein was capable of promoting RDN condensation. Furthermore, the level of suppression of RDN misfolding by the NTS1-targeted fusion protein was underestimated by this assay. More than $40 \%$ of the cells had lost the plasmid expressing the NTS1 gRNA prior to assessing condensation, generating false negatives for suppression. When we normalized to cells in the culture that retained the plasmid, the fraction of cells exhibiting partial or completely suppressed RDN folding was $>40 \%$. These results show that ectopic targeting of Cdc5p specifically to sites of condensin binding in the RDN is capable of significantly replacing cohesin's function in RDN condensation.

\section{Discussion}

In this study, we identified an interactome of early condensation factors (composed of cohesin, condensin, and the polo kinase Cdc5p) that control the establishment of chromosome condensation for the 1.4-Mb RDN locus of budding yeast. Our results suggest that cohesin recruits Cdc5p to the RDN and that Cdc5p actively promotes proper condensation, likely by stimulating condensin activity. When cohesin is absent, Cdc5p stimulates condensin to misfold chromosomes into an irreversibly decondensed state. Our data suggest that productive condensin activity is reliant on cohesin's spatial regulation of Cdc5p. Altogether, our study describes a novel mechanism by which cohesin regulates condensin activity and begins to provide mechanistic insight into how SMC complexes can be spatially and temporally coordinated to achieve their functions.
A

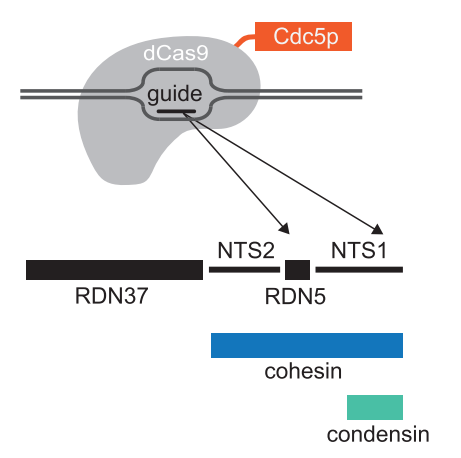

B

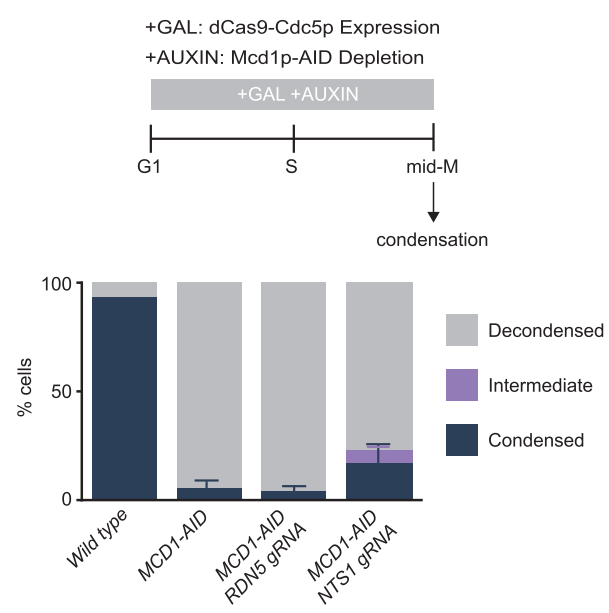

Figure 5. Localization of Cdc5p with CRISPR-Cas9 to the condensin-bound RDN partially restores the RDN condensation defect of cohesin-depleted cells. (A) Galactose addition to the media drives the expression of a catalytically dead dCas9 fused to Cdc5p. Guide RNAs recruit dCas9-Cdc5p fusion protein either to the condensin-bound region NTS1 (RDN5 gRNA) or 500 bp to the left at RDN5 (RDN5 gRNA) where only cohesin is bound. $(B$, top $)$ "Staged mid- $M$ " regimen used to assess condensation in mid-M. Cultures were synchronized in G1 and released in media containing galactose and auxin to drive the expression of dCas9-Cdc5p concomitantly to the depletion of McdlpAID. Media also contained nocodazole to arrest cells in mid-M and assess condensa-

tion. (Bottom) Wild-type, Mcd1p-depleted, Mcd1p-depleted with dCas9-Cdc5p and RDN5 gRNA-expressing, and Mcd1p-depleted with dCas9-Cdc5p and NTS1 gRNA-expressing cells were treated with "staged mid-M" regimen and processed to score RDN condensation. Average of three experiments scoring 100 cells are shown and error bars represent the standard deviation. 


\section{Cdc5p promotes the establishment and maintenance} of condensation prior to anaphase

In this study, we corroborated a previous study that showed that condensation of the RDN in mid-M prior to anaphase requires Cdc5p (polo) kinase as well as CDK kinase (Walters et al. 2014). We show that Cdc5p kinase activity is required for both the establishment and maintenance of RDN condensation in mid-M of budding yeast, likely by modulating condensin. We propose that Cdc5p-dependent phosphorylation of condensin or a condensin regulator is the key step to establish condensation, and that continued phosphorylation is necessary to maintain condensation. We favor condensin as the likely key substrate since a previous study showed that Cdc5p phosphorylates condensin prior to anaphase (St-Pierre et al. 2009). The requirement for CDK and Cdc5p kinases in mid-M RDN condensation might reflect that the binding of polo kinases to their substrates often requires their prior phosphorylation by CDK. Alternatively, CDKs and Cdc5p may regulate different condensin activities like tethering DNA, supercoiling DNA, and extruding DNA loops (Kimura and Hirano 1997; Kimura et al. 2001; Hagstrom et al. 2002; Lavoie et al. 2002; St-Pierre et al. 2009; Ganji et al. 2018). Consistent with this idea, CDK phosphorylation is limited to the Smc4p subunit of condensin (Robellet et al. 2015), while Cdc5p phosphorylates Ycg1p, Ycs4p, and Brnlp subunits (St-Pierre et al. 2009).

\section{Cdc5 recruitment to the condensin-bound region of the RDN modulates condensin to promote proper RDN condensation}

In this study, we provide important clues on how the regulation of condensin by cohesin and Cdc5p is important for the folding of the RDN. First, cohesin is required for the initial recruitment of Cdc5p to chromosomes in mid-M (Mishra et al. 2016; Pakchuen et al. 2016; this study). We show that cohesin recruitment of Cdc5p to chromosomes promotes Cdc5p binding to neighboring condensin. We suggest that cohesin recruitment either directly hands off Cdc5p to neighboring condensin or generates a high local concentration of Cdc5p that promotes its efficient interaction with condensin. By constraining the interaction of Cdc5p with condensin at the RDN, condensin activity is spatially controlled to promote proper RDN folding. In support of this model, we show that cohesin's recruitment function can be replaced by ectopic localization of Cdc5p to the RDN by its fusion with Cas9.

The spatial activation of condensin through a localized kinase adds a critical second feature to the regulation of condensation. Indeed, modulating a kinase's recruitment to its target protein is a very common theme in kinase regulation (Ferrell and Cimprich 2003; Langeberg and Scott 2015). It will be interesting to determine whether the regulation of condensin by CDKs and aurora kinase are also controlled spatially. However, the fact that condensation was not fully restored by the Cas9-Cdc5p fusion suggests that proper $\mathrm{Cdc} 5 \mathrm{p}$ phosphorylation of condensin involves more than simply its general localization to the RDNbound condensin. Both the number of Cdc5p at the RDN and its exact spatial orientation relative to condensin likely differ between the fusion and cohesin bound Cdc5p. Alternatively, cohesin may have a Cdc5p-independent role in condensation. Discovering these features of condensin regulation will require additional studies.

\section{Cohesin localized Cdc5p and RDN misfolding}

Cells lacking cohesin have three phenotypes that inform on the potential molecular function for cohesin-dependent localization of Cdc5p to the RDN. First, the RDN is irreversibly misfolded into a disorganized state (Lavoie et al. 2002; this study). Second, this RDN misfolding is driven by condensin and Cdc5p (Lavoie et al. 2002; this study). Third, Cdc5p phosphorylates Ycglp subunit of condensin with a different phosphopattern (this study). To explain these observations, we suggest that the complex pattern of Cdc5p phosphorylation of condensin is to coordinate condensin's different activities. In wild-type cells, cohesin recruits Cdc5p to condensin, mediating a temporally and spatially controlled Cdc5p phosphorylation of condensin. This regulation of condensin phosphorylation correctly activates the different activities of condensin, resulting in a properly folded RDN. In the absence of cohesin, the delocalized Cdc5p still phosphorylates condensin, but with a different phosphorylation pattern, leading to the uncoordinated control of its activities and RDN misfolding. Inactivating condensin or Cdc5p prevents the formation of the uncoordinated condensin, eliminating RDN misfolding. Identifying these key cohesin-dependent phosphorylation sites will require extensive structural and genetic assays.

In summary, the colocalization of cohesin, polo kinase, and condensin underlies a regulatory pathway that spatially modulates condensin function to generate a thin loop of condensed RDN. The pericentric regions are the only other genomic loci in yeast showing similar colocalization of cohesin, condensin, and polo kinase. We predict that these factors regulate condensation of the pericentric regions to generate thinly condensed structures as they do at the RDN. In yeast, these structures would be difficult to detect cytologically because of the small size of the pericentric regions $(\sim 10 \mathrm{~kb})$. Intriguingly, the pericentric regions are much larger in animals and plants. These regions are visible as thinner areas of the mitotic chromosomes also known as primary constrictions. Constrictions are also observed in the nucleolus organizer regions, the rDNA loci of multicellular organisms. We propose that these constrictions are the result of the same regulatory pathway we described here for the condensation of yeast RDN. Indeed, in vitro condensed chromosomes without cohesin and polo kinase lack constrictions (Shintomi et al. 2015). In vivo, cohesin- and polo kinase-depleted mammalian cells also fail to form primary constrictions (Gandhi et al. 2006). More broadly this regulatory pathway may serve as a general paradigm for how cohesin, condensin, and other SMC complexes communicate to achieve 
proper chromosome structure in other biological processes.

Materials and methods

Yeast strains, growth, and media

All strains used in this study are derived from the A364A background and their genotypes are listed in Table 1. YEP (yeast extract, peptone, and dextrose) medium and YPG (yeast extract, peptone, and galactose) were prepared as described previously (Guacci et al. 1997a). Conditional AID degron strains were grown in YPD or YPG and auxin (3-indoleacetic acid) (Sigma Aldrich I3750) at a final concentration of $500 \mu \mathrm{M}$ to deplete AID-tagged proteins. YPD auxin agar plates were prepared by cooling molten YPD $2 \%$ agar to $55^{\circ} \mathrm{C}$ and supplemented with auxin at a final concentration of $750 \mu \mathrm{M}$. Information regarding the reagents used in this study is in Table 2 .

qPCR oligos

The following oligos were used for qPCR in this study: RDN5-1F (CCGCAACCGATAGTTTAACG), RDN5-1R (TGCTTTTGCT TTTTCACCTG), RDN5-2F (CCCTGCCCTCATATCACCT), RDN5-2R (TGAGTTTCGCGTATGGTCAC), RDN5-3F (CCC
TCATATCACCTGCGTTT), RDN5-3R (AGCACCTGAGTTT CGCGTAT), NTS1-1F (TTATTCCTTCCCGCTTTCCT), NT S1-1R (ATGGAATGGTTGGCGAAGTA), NTS1-2F (TTTACTT CGCCAACCATTCC), NTS1-2R (TGATGATGGCAAGTTC CAGA), NTS1-3F (TCGCCAACCATTCCATATCT), NTS1-3R (TGATGATGGCAAGTTCCAGA), RDN1200F (CAGCTCCGC TTCATTGAATA), RDN1200R (CGAGATTCCCACTGTCC CTA), RDN2100F (CCGGAATCTTAACCGGATTC), RDN210 OR (GAACGGCCTCTAGTGCAGAT), RDN3000F (TTACACCCAAACACTCGCAT), RDN3000R (GACTGAGGACTGCGAC GTAA), RDN5400F (TGAAAACGTCCTTGGCAAAT), RDN54 00R (TGTTCAAAGCAGGCGTATTG), RDN7100F (CCACGATGAGACTGTTCAGG), RDN7100R (GTCGCTAGGTGA TCGTCAGA), RDN7800F (CCGACATTCTGTCCCACATA), RDN7800R (TGGTGCAAAAGACAAATGGA), RDN8200F (TGCGTTTCCGTTAAACTATCG), RDN8200R (GGTGTTAT GGGTGGAGGACA), RDN8600F (CCCTCCATTTCCCTCTC TTC), RDN8600R (GGAATGGTTGGCGAAGTAAA), RDN9 100F (ACTCATGTTTGCCGCTCTG), and RDN9100R (TGCA AAGATGGGTTGAAAGA).

\section{Sister chromatid cohesion}

Sister chromatid cohesion was assessed as previously described (Robison et al. 2018). In brief, in our strains a lacO array is integrated at the LYS4 locus on chromosome IV. These arrays can be

Table 1. Strain table

\begin{tabular}{|c|c|c|}
\hline Strain & Genotype & Reference \\
\hline DK5542 & $\begin{array}{l}\text { MATa MCD1-AID-KANMX6 ADH1-OsTIR1-URA3::ura3-52 lys4::LacO(DK)-NAT trp1-1 GFPLacI- } \\
\text { HIS3:his3-11,15 bar1 leu2-3,112 }\end{array}$ & Eng et al. 2014 \\
\hline TE228 & $\begin{array}{l}\text { MATa PDS5-3V5-AID2-KANMX6 lys4::LacO(DK)-NAT pHIS3-GFP-LacIHIS3::his3-11,15 trp1-1 } \\
\text { ura3-52 }\end{array}$ & Eng et al. 2014 \\
\hline RL06 & $\begin{array}{l}\text { MATa CDC5-3V5-AID2-HPHMX ADH1-OsTIR1-URA3::ura3-52 lys4::LacO(DK)-NAT trp1-1 } \\
\text { GFPLacI-HIS3:his3-11,15 bar1 leu2-3,112 }\end{array}$ & This study \\
\hline RL401 & $\begin{array}{l}\text { MATa BRN1-D375-3V5-AID2-HPHMX trp1 } \triangle:: \text { pGPD1-TIR1-CaTRP1 lys4::LacO(DK)-NAT leu2- } \\
\text { 3,112 pHIS3-GFPLacI-HIS3:his3-11,15 ura3-52 bar1 }\end{array}$ & This study \\
\hline VG3620-4C & $\begin{array}{l}\text { MATa trp1 } \Delta:: \text { pGPD1-TIR1-CaTRP1 lys4::LacO(DK)-NAT leu2-3,112 pHIS3-GFPLacI-HIS3:his3- } \\
\text { 11,15 ura3-52 bar1 }\end{array}$ & $\begin{array}{l}\text { Çamdere et al. } \\
2015\end{array}$ \\
\hline RL404 & $\begin{array}{l}\text { MATa CDC5-3V5-AID2-HPHMX YCG1-S406-6MYC-KANMX ADH1-OsTIR1-URA3::ura3-52 lys4:: } \\
\text { LacO(DK)-NAT trp1-1 GFPLacI-HIS3:his3-11,15 bar1 leu2-3,112 }\end{array}$ & This study \\
\hline RL405 & $\begin{array}{l}\text { MATa MCD1-AID-KANMX6 YCG1-S406-6MYC-HPHMX ADH1-OsTIR1-URA3::ura3-52 lys4:: } \\
\text { LacO(DK)-NAT trp1-1 GFPLacI-HIS3:his3-11,15 bar1 leu2-3,112 }\end{array}$ & This study \\
\hline RL406 & $\begin{array}{l}\text { MATa MCD1-AID-KANMX6 BRN1-D375-3V5-AID2-HPHMX ADH1-OsTIR1-URA3::ura3-52 lys4:: } \\
\text { LacO(DK)-NAT trp1-1 GFPLacI-HIS3:his3-11,15 bar1 leu2-3,112 }\end{array}$ & This study \\
\hline RL407 & $\begin{array}{l}\text { MATa MCD1-AID-KANMX6 CDC5-3V5-AID2-HPHMX ADH1-OsTIR1-URA3::ura3-52 lys4::LacO } \\
\text { (DK)-NAT trp1-1 GFPLacI-HIS3:his3-11,15 bar1 leu2-3,112 }\end{array}$ & This study \\
\hline RL410 & $\begin{array}{l}\text { MATa MCD1-AID-KANMX6 CDC5-3Flag-HPHMX ADH1-OsTIR1-URA3::ura3-52 lys4::LacO(DK)- } \\
\text { NAT trp1-1 GFPLacI-HIS3:his3-11,15 bar1 leu2-3,112 }\end{array}$ & This study \\
\hline RL411 & $\begin{array}{l}\text { MATa BRN1-D375-3V5-AID2-HPHMX CDC5-3Flag-KANMX ADH1-OsTIR1-URA3::ura3-52 lys4:: } \\
\text { LacO(DK)-NAT trp1-1 GFPLacI-HIS3:his3-11,15 bar1 leu2-3,112 }\end{array}$ & This study \\
\hline RL413 & $\begin{array}{l}\text { MATa CDC5-3V5-AID2-HPHMX cdc5-K110A::LEU2 ADH1-OsTIR1-URA3::ura3-52 lys4::LacO } \\
\text { (DK)-NAT trp1-1 GFPLacI-HIS3:his3-11,15 bar1 leu2-3,112 }\end{array}$ & This study \\
\hline RL414 & $\begin{array}{l}\text { MATa YCG1-S406-6MYC-KANMX trp1 } \Delta: \text { :pGPD1-TIR1-CaTRP1 lys4::LacO(DK)-NAT leu2-3,112 } \\
\text { pHIS3-GFPLacI-HIS3:his3-11,15 ura3-52 bar1 }\end{array}$ & This study \\
\hline RL415 & $\begin{array}{l}\text { MATa CDC5-3Flag-HPHMX trp1 } \Delta: \text { :pGPD1-TIR1-CaTRP1 lys4::LacO(DK)-NAT leu2-3,112 pHIS3- } \\
\text { GFPLacI-HIS3:his3-11,15 ura3-52 bar1 }\end{array}$ & This study \\
\hline RL416 & MATa MCD1-AID-KANMX6 TIR1-LEU2 pHIS3-GFPLacI-HIS3:his3-11,15 ura3-52 bar1 & This study \\
\hline RL417 & $\begin{array}{l}\text { MATa MCD1-AID-KANMX6 pGAL-dCas9-CDC5-3Flag-TRP1:trp1 } \Delta \text { (RDN5 2u URA3) TIR1-LEU2 } \\
\text { pHIS3-GFPLacI-HIS3:his3-11,15 ura3-52 bar1 }\end{array}$ & This study \\
\hline RL418 & $\begin{array}{l}\text { MATa MCD1-AID-KANMX6 pGAL-dCas9-CDC5-3Flag-TRP1:trp1 } \Delta \text { (NTS1 2u URA3) TIR1-LEU2 } \\
\text { pHIS3-GFPLacI-HIS3:his3-11,15 ura3-52 bar1 }\end{array}$ & This study \\
\hline RL419 & MATa CDC5-AID-HPHMX TIR1-LEU2 pHIS3-GFPLacI-HIS3:his3-11,15 ura3-52 bar1 & This study \\
\hline
\end{tabular}


Table 2. Reagents

\begin{tabular}{|c|c|c|c|c|}
\hline $\begin{array}{l}\text { Reagent type or } \\
\text { resource }\end{array}$ & Designation & $\begin{array}{l}\text { Source or } \\
\text { reference }\end{array}$ & Identifiers & Additional information \\
\hline $\begin{array}{l}\text { Genetic reagent }(S . \\
\text { cerevisiae) }\end{array}$ & NCBI Taxon 4932 & This paper & Yeast strains & Supplemental File 1 \\
\hline Primers & DNA primer & IDT & & \\
\hline Antibody & Rabbit polyclonal anti-Mcdlp & $\begin{array}{l}\text { V. Guacci (via } \\
\text { Covance) }\end{array}$ & RbaMcd1p (555) & $\begin{array}{l}\text { Western blot }(1: 10,000) ; \\
\text { ChIP-seq }(1: 1000)\end{array}$ \\
\hline Antibody & Rabbit polyclonal anti-Mcdlp & $\begin{array}{l}\text { V. Guacci (via } \\
\text { Covance) }\end{array}$ & RbaPds5p (556) & $\begin{array}{l}\text { Western blot }(1: 20,000) \\
\text { ChIP-seq }(1: 1000)\end{array}$ \\
\hline Antibody & Mouse monoclonal anti-MYC & Roche & $\begin{array}{r}\text { MaMYC (9E10), } \\
116666006001\end{array}$ & $\begin{array}{l}\text { Western blot }(1: 10,000) \\
\text { ChIP-seq }(1: 1000)\end{array}$ \\
\hline Antibody & Mouse monoclonal nti-Flag & Sigma Aldrich & MaFlag, F3165 & $\begin{array}{l}\text { Western blot }(1: 10,000) \\
\text { ChIP-seq }(1: 1000)\end{array}$ \\
\hline Antibody & Mouse monoclonal anti-V5 & Invitrogen & MaV5, 46-0705 & $\begin{array}{l}\text { Western blot }(1: 10,000) \\
\text { ChIP-seq }(1: 1000)\end{array}$ \\
\hline Antibody & Goat polyclonal HRP antirabbit & Biorad & $170-6515$ & Western blot $(1: 10,000)$ \\
\hline Antibody & $\begin{array}{l}\text { Goat polyclonal HRP } \\
\text { antimouse }\end{array}$ & Biorad & $170-6516$ & Western blot $(1: 10,000$ \\
\hline Antibody & Rabbit polyclonal anti-Tub2p & $\begin{array}{l}\text { P. Meluh (via } \\
\text { Covance) }\end{array}$ & RbaTUB2 & Western blot $(1: 40,000)$ \\
\hline Dynabeads & Protein A & Invitrogen & $10002 \mathrm{D}$ & $100 \mu \mathrm{L}$ per IP \\
\hline Chemical compound & Auxin (3-indole acetic acid) & Sigma Aldrich & C9911 & $\begin{array}{l}500 \mu \mathrm{M} \text { for liquid (dissolved } \\
\text { in ethanol) }\end{array}$ \\
\hline Chemical compound & a Factor & Sigma Aldrich & $\alpha \mathrm{F}$ (a factor) $\mathrm{T} 6901$ & $10 \mu \mathrm{M}$ \\
\hline Chemical compound & Nocodazole & Sigma Aldrich & Nz M1404 & Double check \\
\hline Reagent & Bioruptor beads & Diagenode & C01020031 & $200 \mu \mathrm{L}$ per sample \\
\hline $\begin{array}{l}\text { Sequencing and } \\
\text { library kits }\end{array}$ & $\begin{array}{l}\text { Accel-NGS 1S Plus DNA } \\
\text { library kit }\end{array}$ & Swift Bioscience & 10024 & \\
\hline
\end{tabular}

visualized by binding of GFP-lacI, which is integrated at the HIS3 locus. To assess cohesion, cells were grown to early log phase $\left(\mathrm{OD}_{600} 0.1-0.2\right)$ overnight at $23^{\circ} \mathrm{C}$ and arrested in G1 using the pheromone $\alpha$ factor at a concentration of $10 \mu \mathrm{M}$ (Sigma Aldrich T6901-5MG). If indicated, auxin was added to a final concentration of $500 \mu \mathrm{M}$ to deplete AID-tagged proteins after a 2.5-h arrest. Cells were released from G1 by washing with YPD containing auxin (if indicated) and $0.15 \mathrm{mg} / \mathrm{mL}$ pronase $\mathrm{E}$ (Sigma Aldrich) three times, and once with YPD plus auxin. After the last wash, cells were resuspended in YPD medium containing auxin and $15 \mu \mathrm{g} /$ $\mathrm{mL}$ nocodazole (Sigma Aldrich). Cultures were then once again incubated for $3 \mathrm{~h}$ at $23^{\circ} \mathrm{C}$. One milliliter of these cells was then fixed in $70 \%$ ethanol and stored at $4^{\circ} \mathrm{C}$ until ready for visualization. Cohesion was assessed by scoring the number of GFP-lacI foci in each cell on an Axioplan2 microscope (Zeiss) using the 100× objective (numerical aperture 1.40) equipped with a Quantix charge-coupled camera (Photometrics). For time-course experiments, $1 \mathrm{~mL}$ of cells was collected every $15 \mathrm{~min}$ after release from $a$ factor.

\section{Assessing condensation at the RDN locus}

Cells were grown as described for the sister chromatid cohesion assay. After $3 \mathrm{~h}$ in nocodazole, cells were fixed, spheroplasted, and prepared for FISH as described previously (Guacci et al. 1994). DNA masses were then visualized with DAPI (4',6- diamidino-2-phenylindole) and imaged as described above. RDN morphology was scored as condensed "looped" or decondensed "puffy" as described previously (Guacci et al. 1994).

\section{Maintenance assay}

For maintenance assays, cells were grown to early log phase at $23^{\circ} \mathrm{C}$ and arrested in mid-M using $15 \mu \mathrm{g} / \mathrm{mL}$ nocodazole. For
AID-tagged proteins, auxin was added after synchronous mid-M arrest, $2 \mathrm{~h}$ after nocodazole addition. Cells were collected after $1 \mathrm{~h}$ of auxin addition for condensation assay.

\section{'Add later' time course}

Cells were grown as described in the sister chromatid cohesion assay to get synchronous population of cells arrested in mid-M depleted for the AID-tagged protein(s). To restore the presence of the AID-tagged proteins, these cells were washed three times in YPD medium containing nocodazole and grown in media containing nocodazole for $2 \mathrm{~h}$. Samples were collected after $2 \mathrm{~h}$ to assess condensation state and protein levels by TCA extraction followed by Western.

\section{TCA protein extraction and Western blotting}

To assess protein level, cells were collected, pelleted, washed with $1 \times$ PBS and frozen at $-80^{\circ} \mathrm{C}$. Frozen pellets were resuspended in $200 \mu \mathrm{L}$ of $20 \%$ TCA and broken for 40 sec using a FastPrep-24 $5 \mathrm{G}$ instrument $(6.0 \mathrm{~m} / \mathrm{sec}$ each) (MP Bio). Lysates were diluted with $1 \mathrm{~mL}$ of $5 \%$ TCA and spun at $14,000 \mathrm{rpm}$ for $10 \mathrm{~min}$ at $4^{\circ}$ C. Pellets were resuspended in $2 \times$ Laemmli buffer, boiled for 7 min, and spun. Cleared lysates were used for Western blotting on $6 \%$ SDS-PAGE gels. Phos-tag gels were prepared, run, and transferred following the manufacturer protocol (Alphalabs).

\section{Chromatin immunoprecipitation (ChIP) and sequencing}

Cells were grown and arrested at $30^{\circ} \mathrm{C}$ as described for assessing condensation. Mid-M cells (60OD) were collected, fixed for $1 \mathrm{~h}$ with $1 \%$ formaldehyde, quenched with $5 \mathrm{mM}$ glycine, and processed for ChIP as described previously (Robison et al. 2018), 
with the following modifications. Frozen cell pellets were disrupted three times using a FastPrep-24 5G instrument for 40 sec $(6.0 \mathrm{~m} / \mathrm{sec}$ each) (MP Bio). Chromatin was sheared $10 \times 30$ sec on/30 sec off with a Bioruptor Pico (Diagenode). For ChIPqPCR, 1/20 of sheared and cleared lysate was reserved as input. Chromatin extracts were incubated with $4 \mu \mathrm{L}$ of the following antibodies monoclonal mouse anti-MYC (Roche), monoclonal mouse anti-Flag (Sigma Aldrich), polyclonal rabbit anti-Pds5p (Covance Biosciences), or polyclonal rabbit anti-Mcdlp (Covance Biosciences) overnight at $4^{\circ} \mathrm{C}$. Antibody-bound lysates were incubated with $120 \mu \mathrm{L}$ Protein A dynabeads (Invitrogen 10001D) for 1 h. Following ChIP, the library was prepared using the Accel-NGS 1S Plus DNA library kit (Swift Bioscience) following the manufacturer's protocol. Libraries were sequenced using Illumina Hiseq 4000. The sequencing files were aligned to the SacCer3 yeast genome using Bowtie 2 tool and bigwig files were normalized for number of reads.

Accession numbers

The data generated are available at Gene Expression Omnibus (GEO) under accession number GSE147290

\section{dCas9-Cdc5p experiments}

Cells were grown in $5 \mathrm{~mL}$ of -URA medium until ready to be used to maintain plasmid. Cells were grown to early log phase $\left(\mathrm{OD}_{600}\right.$ 0.2 ) overnight at $23^{\circ} \mathrm{C}$ and arrested in G1 using the pheromone $\alpha$ factor at a concentration of $10 \mu \mathrm{M}$ (Sigma Aldrich T6901-5MG) in YEP $+2 \%$ lactic acid medium. After $2.5 \mathrm{~h}$ of growth in a factor, auxin was added to a final concentration of $500 \mu \mathrm{M}$ to deplete AID-tagged proteins and galactose was added to a final concentration of $2 \%$ to induce expression of pGal-dCas9-Cdc5-3Flag construct. After $1 \mathrm{~h}$, cells were released from G1 by washing with YEP $+2 \%$ galactose medium containing auxin and $0.15 \mathrm{mg} / \mathrm{mL}$ pronase E (Sigma Aldrich) three times, and once with YEP + $2 \%$ galactose plus auxin. After the last wash, cells were resuspended in YEP $+2 \%$ galactose medium containing auxin and $15 \mu \mathrm{g} / \mathrm{mL}$ nocodazole (Sigma Aldrich). Cultures were then once again incubated for $3 \mathrm{~h}$ at $23^{\circ} \mathrm{C}$. One milliliter of these cells was then processed as described above in "Assessing Condensation at the RDN Locus."

\section{Acknowledgments}

We thank Thomas Eng and Vincent Guacci for building some of the strains used. We thank Elcin Unal for sharing the dCas9 plasmid, and Fred Winston for critical reading of the manuscript. We thank the Koshland laboratory and the Unal laboratory for input and discussions. This work was funded by National Science Foundation Graduate Research Fellowship Program grant to R.L. and National Institutes of Health grant 1R35 GM-11818901 to D.E.K.

Author contributions: R.L. did the genetics and cytological studies on cohesin, condensin, and polo kinase. L.C. performed the ChIP-seq and ChIP-qPCR analysis. All three authors participated in designing the experiments and drafting the manuscript.

\section{References}

Archambault V, Lépine G, Kachaner D. 2015. Understanding the Polo Kinase machine. Oncogene 34: 4799-4807. doi:10.1038/ onc.2014.451
Çamdere G, Guacci V, Stricklin I, Koshland D. 2015. The ATPases of cohesin interface with regulators to modulate cohesin-mediated DNA tethering. Elife 4: e11315. doi:10 $.7554 /$ eLife. 11315

D'Ambrosio C, Schmidt CK, Katou Y, Kelly G, Itoh T, Shirahige $\mathrm{K}$, Uhlmann F. 2008. Identification of cis-acting sites for condensin loading onto budding yeast chromosomes. Genes Dev 22: 2215-2227. doi:10.1101/gad.1675708

Ding DQ, Sakurai N, Katou Y, Itoh T, Shirahige K, Haraguchi T, Hiraoka Y. 2006. Meiotic cohesins modulate chromosome compaction during meiotic prophase in fission yeast. I Cell Biol 174: 499-508. doi:10.1083/jcb.200605074

Eng T, Guacci V, Koshland D. 2014. ROCC, a conserved region in cohesin's Mcdl subunit, is essential for the proper regulation of the maintenance of cohesion and establishment of condensation. Mol Biol Cell 25: 2351-2364. doi:10.1091/mbc.e14-040929

Ferrell JE Jr, Cimprich KA. 2003. Enforced proximity in the function of a famous scaffold. Mol Cell 11: 289-291. doi:10.1016/ S1097-2765(03)00055-8

Ganji M, Shaltiel IA, Bisht S, Kim E, Kalichava A, Haering CH, Dekker C. 2018. Real-time imaging of DNA loop extrusion by condensin. Science 360: 102-105. doi:10.1126/science .aar7831

Gandhi R, Gillespie PJ, Hirano T. 2006. Human Wapl is a cohesinbinding protein that promotes sister-chromatid resolution in mitotic prophase. Curr Biol 16: 2406-2417. doi:10.1016/j .cub.2006.10.061

Glynn EF, Megee PC, Yu HG, Mistrot C, Unal E, Koshland DE, DeRisi JL, Gerton JL. 2004. Genome-wide mapping of the cohesin complex in the yeast Saccharomyces cerevisiae. PLOS Biol 2: E259. doi:10.1371/journal.pbio.0020259

Guacci V, Koshland D. 2012. Cohesin-independent segregation of sister chromatids in budding yeast. Mol Biol Cell 23: 729-739. doi:10.1091/mbc.e11-08-0696

Guacci V, Hogan E, Koshland D. 1994. Chromosome condensation and sister chromatid pairing in budding yeast. I Cell Biol 125: 517-530. doi:10.1083/jcb.125.3.517

Guacci V, Hogan E, Koshland D. 1997a. Centromere position in budding yeast: evidence for anaphase A. Mol Biol Cell 8: 957-972. doi:10.1091/mbc.8.6.957

Guacci V, Koshland D, Strunnikov A. 1997b. A direct link between sister chromatid cohesion and chromosome condensation revealed through the analysis of MCD1 in S. cerevisiae. Cell 91: 47-57. doi:10.1016/S0092-8674(01)80008-8

Hagstrom KA, Holmes VF, Cozzarelli NR, Meyer BJ. 2002. C. elegans condensin promotes mitotic chromosome architecture, centromere organization, and sister chromatid segregation during mitosis and meiosis. Genes Dev 16: 729-742. doi:10 $.1101 /$ gad.968302

Hassler M, Shaltiel IA, Haering CH. 2018. Towards a unified model of SMC complex function. Curr Biol 28: R1266R1281. doi:10.1016/j.cub.2018.08.034

Jin H, Guacci V, Yu HG. 2009. Pds5 is required for homologue pairing and inhibits synapsis of sister chromatids during yeast meiosis. J Cell Biol 186: 713-725. doi:10.1083/jcb.200810107

Kimura K, Hirano T. 1997. ATP-dependent positive supercoiling of DNA by $13 \mathrm{~S}$ condensin: a biochemical implication for chromosome condensation. Cell 90: 625-634. doi:10.1016/S00928674(00) 80524-3

Kimura K, Hirano M, Kobayashi R, Hirano T. 1998. Phosphorylation and activation of $13 \mathrm{~S}$ condensin by $\mathrm{Cdc} 2$ in vitro. Science 282: 487-490. doi:10.1126/science.282.5388.487 
Kimura K, Cuvier O, Hirano T. 2001. Chromosome condensation by a human condensin complex in Xenopus egg extracts. I Biol Chem 276: 5417-5420. doi:10.1074/jbc.C000873200

Laloraya S, Guacci V, Koshland D. 2000. Chromosomal addresses of the cohesin component Mcd1p. J Cell Biol 151: 1047-1056. doi:10.1083/jcb.151.5.1047

Langeberg LK, Scott JD. 2015. Signalling scaffolds and local organization of cellular behaviour. Nat Rev Mol Cell Bio 16: 232244. doi:10.1038/nrm3966

Lavoie BD, Hogan E, Koshland D. 2002. In vivo dissection of the chromosome condensation machinery: reversibility of condensation distinguishes contributions of condensin and cohesin. J Cell Biol 156: 805-815. doi:10.1083/jcb.200109056

Lavoie BD, Hogan E, Koshland D. 2004. In vivo requirements for rDNA chromosome condensation reveal two cell-cycle-regulated pathways for mitotic chromosome folding. Genes Dev 18: 76-87. doi:10.1101/gad.1150404

Leonard J, Sen N, Torres R, Sutani T, Jarmuz A, Shirahige K, Aragón L. 2015. Condensin relocalization from centromeres to chromosome arms promotes Top2 recruitment during anaphase. Cell Rep 13: 2336-2344. doi:10.1016/j.celrep.2015.11 .041

Losada A, Hirano M, Hirano T. 1998. Identification of Xenopus SMC protein complexes required for sister chromatid cohesion. Genes Dev 12: 1986-1997. doi:10.1101/gad.12.13.1986

Michaelis C, Ciosk R, Nasmyth K. 1997. Cohesins: chromosomal proteins that prevent premature separation of sister chromatids. Cell 91: 35-45. doi:10.1016/S0092-8674(01)80007-6

Mishra PK, Ciftci-Yilmaz S, Reynolds D, Au WC, Boeckmann L, Dittman LE, Jowhar Z, Pachpor T, Yeh E, Baker RE, et al. 2016. Polo kinase Cdc5 associates with centromeres to facilitate the removal of centromeric cohesin during mitosis. Mol Biol Cell 27: 2286-2300. doi:10.1091/mbc.E16-01-0004

Nishimura K, Fukagawa T, Takisawa H, Kakimoto T, Kanemaki M. 2009. An auxin-based degron system for the rapid depletion of proteins in nonplant cells. Nat Methods 6: 917-922. doi:10.1038/nmeth.1401

Noble D, Kenna MA, Dix M, Skibbens RV, Ünal E, Guacci V. 2006. Intersection between the regulators of sister chromatid cohesion establishment and maintenance in budding yeast indicates a multi-step mechanism. Cell Cycle 5: 2528-2536. doi:10.4161/cc.5.21.3405

Novak I, Wang H, Revenkova E, Jessberger R, Scherthan H, Höög C. 2008. Cohesin Smc1 $\beta$ determines meiotic chromatin axis loop organization. I Cell Biol 180: 83-90. doi:10.1083/jcb .200706136

Pakchuen S, Ishibashi M, Takakusagi E, Shirahige K, Sutani T. 2016. Physical association of Saccharomyces cerevisiae Polo-like kinase Cdc5 with chromosomal cohesin facilitates DNA damage Response. I Biol Chem 291: 17228-17246. doi:10.1074/jbc.M116.727438
Robellet X, Thattikota Y, Wang F, Wee TL, Pascariu M, Shankar S, Bonneil E, Brown CM, D'Amours D. 2015. A high-sensitivity phospho-switch triggered by Cdk1 governs chromosome morphogenesis during cell division. Genes Dev 29: 426-439. doi:10.1101/gad.253294.114

Robison B, Guacci V, Koshland D. 2018. A role for the Smc3 hinge domain in the maintenance of sister chromatid cohesion. Mol Biol Cell 29: 339-355. doi:10.1091/mbc.E17-08-0511

Rolef Ben-Shahar T, Heeger S, Lehane C, East P, Flynn H, Skehel M, Uhlmann F. 2008. Eco1-dependent cohesin acetylation during establishment of sister chromatid cohesion. Science 321: 563-566. doi:10.1126/science.1157774

Rossio V, Galati E, Ferrari M, Pellicioli A, Sutani T, Shirahige K, Lucchini G, Piatti S. 2010. The RSC chromatin-remodeling complex influences mitotic exit and adaptation to the spindle assembly checkpoint by controlling the Cdc14 phosphatase. I Cell Biol 191: 981-997. doi:10.1083/jcb.201007025

Shintomi K, Takahashi TS, Hirano T. 2015. Reconstitution of mitotic chromatids with a minimum set of purified factors. Nat Cell Biol 17: 1014-1023. doi:10.1038/ncb3187

Sonoda E, Matsusaka T, Morrison C, Vagnarelli P, Hoshi O, Ushiki T, Nojima K, Fukagawa T, Waizenegger IC, Peters JM, et al. 2001. Scc1/Rad21/Mcd1 is required for sister chromatid cohesion and kinetochore function in vertebrate cells. Dev Cell 1: 759-770. doi:10.1016/S1534-5807(01)00088-0

St-Pierre J, Douziech M, Bazile F, Pascariu M, Bonneil E, Sauvé V, Ratsima H, D'Amours D. 2009. Polo kinase regulates mitotic chromosome condensation by hyperactivation of condensin DNA supercoiling activity. Mol Cell 34: 416-426. doi:10 .1016/j.molcel.2009.04.013

Straight AF, Belmont AS, Robinett CC, Murray AW. 1996. GFP tagging of budding yeast chromosomes reveals that proteinprotein interactions can mediate sister chromatid cohesion. Curr Biol 6: 1599-1608. doi:10.1016/S0960-9822(02)70783-5

Teytelman L, Thurtle DM, Rine J, van Oudenaarden A. 2013. Highly expressed loci are vulnerable to misleading ChIP localization of multiple unrelated proteins. Proc Nat1 Acad Sci 110: 18602-18607. doi:10.1073/pnas.1316064110

Ünal E, Heidinger-Pauli JM, Kim W, Guacci V, Onn I, Gygi SP, Koshland DE. 2008. A molecular determinant for the establishment of sister chromatid cohesion. Science 321: 566569. doi:10.1126/science.1157880

Vas AC, Andrews CA, Kirkland Matesky K, Clarke DJ. 2007. In vivo analysis of chromosome condensation in Saccharomyces cerevisiae. Mol Biol Cell 18: 557-568. doi:10.1091/mbc.e0605-0454

Walters AD, May CK, Dauster ES, Cinquin BP, Smith EA, Robellet X, D'Amours D, Larabell CA, Cohen-Fix O. 2014. The yeast polo kinase Cdc5 regulates the shape of the mitotic nucleus. Curr Biol 24: 2861-2867. doi:10.1016/j.cub.2014.10.029 


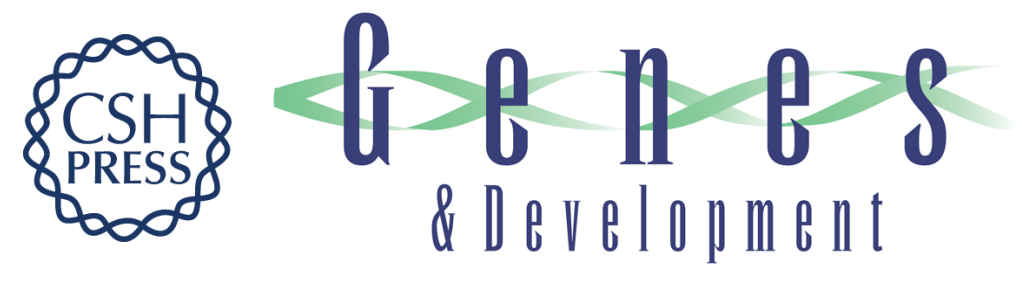

\section{The spatial regulation of condensin activity in chromosome condensation}

Rebecca Lamothe, Lorenzo Costantino and Douglas E. Koshland

Genes Dev. 2020, 34: originally published online April 30, 2020

Access the most recent version at doi:10.1101/gad.335471.119

\section{Supplemental http://genesdev.cshlp.org/content/suppl/2020/04/30/gad.335471.119.DC1 Material}

References This article cites 43 articles, 24 of which can be accessed free at: http://genesdev.cshlp.org/content/34/11-12/819.full.html\#ref-list-1

Creative This article is distributed exclusively by Cold Spring Harbor Laboratory Press for the first Commons six months after the full-issue publication date (see

License http://genesdev.cshlp.org/site/misc/terms.xhtml). After six months, it is available under a Creative Commons License (Attribution-NonCommercial 4.0 International), as described at http://creativecommons.org/licenses/by-nc/4.0/.

Email Alerting Receive free email alerts when new articles cite this article - sign up in the box at the top Service right corner of the article or click here.

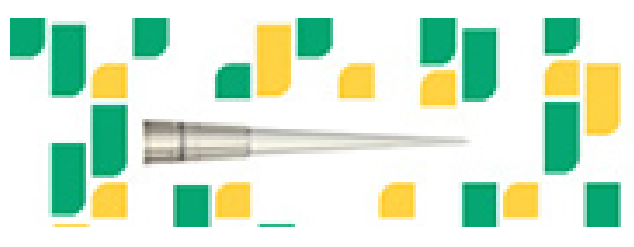

Focused on your science. 